\title{
Staphylococcus aureus in a neonatal care center: methicillin-susceptible strains should be a main concern
}

\author{
Sara Romano-Bertrand ${ }^{1,2^{*}}$, Anne Filleron ${ }^{1,3}$, Renaud Mesnage ${ }^{4}$, Anne Lotthé2 ${ }^{2}$ Marie Noëlle Didelot ${ }^{5}$, Lydie Burgel ${ }^{2}$, \\ Estelle Jumas Bilak ${ }^{1,2}$, Gilles Cambonie ${ }^{4}$ and Sylvie Parer ${ }^{2}$
}

\begin{abstract}
Background: In the context of a methicillin-susceptible Staphylococcus aureus (MSSA) outbreak, we aimed to improve our knowledge of S. aureus (SA) epidemiology in the neonatal care center (NCC) of a tertiary care teaching hospital.

Methods: We performed a complete one-year review of SA carrier, colonized or infected patients. Monthly prevalence and incidence of SA intestinal carriage, colonization and infection were calculated and the types of infection analysed. During the MSSA outbreak, strains were studied for antimicrobial resistance, content of virulence genes and comparative fingerprint in Pulsed-Field Gel Electrophoresis. Hand hygiene and catheter-related practices were assessed by direct observational audits. Environmental investigation was performed in search of a SA reservoir.

Results: Epidemiological analyses showed 2 or 3 prevalence peaks on a background of SA endemicity. In the NCC, during 2009, overall MSSA prevalence did not decrease below 5.5\%, while mean MRSA prevalence was about $1.53 \%$. Analysis of infection cases revealed that the outbreak corresponded to the emergence of catheter-related infections and was probably related to the relaxation in infection control practices in a context of high colonization pressure. Health care workers' white coats appeared as a potential environmental reservoir that could perpetuate SA circulation in the ward.
\end{abstract}

Conclusion: This report emphasizes the importance of integrating MSSA along with methicillin-resistant SA in a program of epidemiological surveillance in the NCC.

Keywords: Staphylococcus aureus, Prevalence, Catheter-related infections, White coat contamination, Methicillin-susceptible Staphylococcus aureus

\section{Background}

The high incidence of hospital-acquired infections (HAIs) in neonatal intensive care units (NICUs) is related to the immaturity of patients who are also subjected to many invasive procedures. Coagulase negative staphylococci (CoNS) and Staphylococcus aureus (SA) are the main and often sole bacteria colonizing the digestive tract of

\footnotetext{
*Correspondence: sara.romano-bertrand@univ-montp1.fr

'Université Montpellier 1, UMR 5119, Equipe Pathogènes et Environnements,

U.F.R. des Sciences Pharmaceutiques et Biologiques, 15, Avenue Charles Flahault, BP 14491, 34093 Montpellier Cedex 5, France

${ }^{2}$ Centre Hospitalier Régional Universitaire de Montpellier, Hôpital La Colombière, Service d'Hygiène Hospitalière, 39 avenue Charles Flahault, 34295 Montpellier Cedex 5, France

Full list of author information is available at the end of the article
}

low birth-weight infants during the 3 first weeks of life [1]. Furthermore, CoNS and SA are responsible for most infections in hospitalized preterm infants [2-4].

As in other hospital units, methicillin-resistant S. aureus (MRSA) outbreaks have often been reported in NICUs [5-8] whereas, at first glance, methicillin-susceptible $S$. aureus (MSSA) outbreaks seem less frequent. Indeed, a PubMed database search returns 1108 papers for "MRSA outbreak" versus 52 for "MSSA outbreak" (January 2014). In second analysis, the scarcity of MSSA outbreaks could be due to a bias in detection or reporting, MRSA being one of the most threatening pathogens as well as the principal indicator of nosocomial risk. Patients' screening and outbreak alert systems in most hospitals focus on MRSA, 
while MSSA infections are generally treated piecemeal with little or no insight into molecular typing and epidemiology. However, a study of $358 \mathrm{~S}$. aureus strains $(2,007,681$ days of hospitalization in 32 healthcare institutions) showed there is a significant increase of bloodstream HAIs largely due to MSSA strains $[9,10]$.

The published MSSA outbreaks concerned merely NICUs [11-13], burns units [14] and multi-resistant MSSA [14,15]. The spread of MSSA clonal strains in NICUs seems to be very successful. For instance, in a 5-year outbreak affecting 202 neonates, classical control measures failed to end the outbreak [13] but atypical reservoirs near the patient, such as skin protectant [13] and ultrasound gel [12] were found. Intestinal carriage of SA seems to be neglected in NICUs, although it frequently occurs in infants $[1,16,17]$. Furthermore, it is associated with a high risk of skin colonization which can in turn increase the risk of infections, environmental contamination and cross transmission [17].

The aim of this study is to describe the epidemiology of SA in the neonatal care center (NCC) of the Montpellier Academic Hospital. An increase of MSSA colonization and infection cases in the NCC led to a complete investigation: (i) analysis of cases, (ii) assessment of hygiene practices (hand hygiene and catheter-related care) (iii) search of SA environmental reservoir, (iv) molecular typing. The outbreak was confirmed and related to the spread of a common strain with a probable environmental reservoir.

\section{Methods}

\section{Settings, patients and infection surveillance policies}

The NCC of Montpellier is organized in 3 sectors: the paediatric reanimation and intensive care unit or PRICU (14 beds in 9 boxes for neonates including very preterm neonates and 6 beds in 5 rooms for infants), the NICU (24 beds in 10 boxes), and a mother-cum-child or "kangaroo" ward ( 9 beds in individual rooms, and 3 beds in a nursery).

Hospitalized patients in the PRICU are low birth-weight $(<1500 \mathrm{~g})$ preterm infants and newborns aged $<1$ month with diseases or unstable states. The PRICU also hosts newborns having surgery and older children requiring intensive care. Newborns are transferred to the NICU once their clinical state is stabilised or improved. As soon as their condition allows, patients are transferred to the kangaroo ward before returning home.

The medical and paramedical teams from the PRICU, the NICU and the kangaroo ward, a microbiologist and a member of the infection control (IC) team meet weekly to discuss HAI cases, differentiating true HAIs from bacterial carriage or colonization by confronting microbiological data, biological and clinical contexts and initiation of antimicrobial treatment [18]. Infants are considered as colonized if a positive culture is obtained from a non-sterile site, and infected if a pathogen is isolated from a normally sterile site or if cultures are obtained for clinical purposes. In addition, in the NICU sector, the digestive carriage of SA and multi-drug resistant bacteria (MDRB) is screened for each patient upon admission and once a week thereafter. A positive culture from a digestive sample is considered as SA carriage [17]. Stool samples are cultured to determine methicillin and ceftazidime resistance in staphylococci and gram-negative bacilli, respectively. MDRB detection leads to supplementary infection control measures to prevent cross-contamination among patients, and to further environmental investigations. A real-time surveillance is implemented by a daily account of all newly MDRB colonized or infected patients by the microbiologist, using an antibiotic resistance information system (Sirweb", i2a, Montpellier, France). Surveillance data is transmitted to the IC team via the hospital information system. This automatic surveillance system operates for MDRB only; hence outbreaks involving susceptible micro-organisms can be detected only through clinical observation of an increased number of cases over a given time span.

\section{Outbreak investigation of MSSA infections}

This clinical surveillance thus detected an increase in HAIs involving MSSA, leading to an outbreak investigation by the IC team including analysis of patients' medical records, environmental investigation, and assessment of healthcare practices. Clinical strains were tested for their antimicrobial susceptibility by disk diffusion assay according to the French Committee for Antimicrobial Susceptibility Testing (Members of the Société Française de Microbiologie Committee, 2003). After extraction of the DNA performed according to Predari et al. [19], isolates were screened for genes encoding staphylococcal enterotoxins A (sea), toxic shock syndrome toxin 1 (tst), Panton-Valentine leukocidin (PVL; luk-PV) [2-4]. Clinical MSSA strains were explored by genomotyping to determine genetic links between them. For this purpose, intact genomic DNA was extracted in agarose plugs and digested by the endonuclease SmaI, as described [20]. Macrorestriction fragments were separated by pulsed-field gel electophoresis (PFGE) by a ramp of pulses of 20s to $5 \mathrm{~s}$ at $6 \mathrm{~V} / \mathrm{min}$ during $24 \mathrm{~h}$ on CHEF-DRII apparatus (Biorad).

Dry and humid surfaces were sampled, as well as health-care workers' clothes (i.e.: white over-gowns used for the manipulation of central venous lines, especially the front part which comes into contact with patients); the cotton swabs used for sampling served to inoculate trypticase-soy, Chapman and McConkey agar plates (bioMérieux, France) which were incubated at $37^{\circ} \mathrm{C}$ for $48 \mathrm{~h}$. Water was sampled at different points of use in the units in $250 \mathrm{~mL}$ sterile bottles containing sodium thiosulfate for chloride inhibition. Water samples were 
passed through a $0.22 \mu \mathrm{m}$ nylon filters, which were then incubated at $30^{\circ} \mathrm{C}$ during $48 \mathrm{~h}$ on Chapman agar. Healthcare practices in the NCC were audited by direct observation of hand hygiene opportunities (a minimum of 30 observations) and central venous catheter manipulations (a minimum of 5 observations).

\section{Epidemiology}

Monthly prevalence and incidence of SA colonizations and infections were calculated for each sector, as well as the prevalence of MSSA and MRSA for all the NCC. Prevalence was defined as number of patients with SA per total number of hospitalized patients in the month, reflecting the endemicity of SA in the NCC. The incidence was the ratio of new cases on new admissions during the month. Duplicate cases in a same sector (repeat admission) or between sectors (transfer of a previously known case) were excluded from prevalence and incidence calculations.

\section{Ethical considerations}

We studied bacterial isolates obtained during the daily care of preterm infants in our NICU. Therefore, this observational study was fully in line with the routine care of preterm infants and did not require the agreement of the ethical committee of our institution.

\section{Results}

\section{SA epidemiology in the NCC}

One hundred and thirty nine patients admitted to the NCC were included in the epidemiological study. Distribution of carriage, colonization and infection cases for each sector is summarized in Figure 1. Fifty-four patients were carriers, colonized or infected by SA, 22 in the summer period (4, 6, 4 and 8 respectively in June, July, August and September). In the NICU and kangaroo ward, most
SA isolates were involved in carriage with a low frequency of colonization and/or infection. By contrast, patients in the PRICU with more instable clinical status were more frequently colonized and/or infected.

Distribution of the different types of SA positive clinical samples corresponding to colonization and/or infection by care sector is summarized in Table 1. Most of them occurred in the PRICU (70.9\% of all positive samples), followed by the NICU (23.3\%) and the kangaroo ward (5.8\%). Respiratory samples were predominant in the NCC, overall representing 52.3\% of all clinical samples, especially in the PRICU (63.9\%). Ophthalmic samples were the second most frequent with about $17.5 \%$, followed by blood cultures (10\%) and catheter samples (7\%). By care sector, ophthalmic colonization and infections predominated in the NICU, and came equal with respiratory samples ( $40 \%$ each) in the kangaroo ward. SA positive catheter samples and blood cultures were mainly present in the PRICU ( 8 of the 10 samples), 2 other cases were found in the NICU, and none in the kangaroo ward where patients are less often perfused.

The evolution of SA monthly prevalence for each sector is represented on Figure 2. In the NICU, where digestive carriage of SA is routinely screened, average prevalence reached $12.9 \%$, with increasing rates in March (20\%), August (17\%) and November (22\%). In other sectors, because of the absence of systematic screening, prevalence rates obtained were certainly underestimated and did not reflect the real presence of SA. In the PRICU, average rates were around $5.5 \%$ and ranged generally between 5 and 10\%, except in November and December when they fell to $3.1 \%$ and $1.1 \%$ respectively. In the kangaroo ward, rates were more variable, roughly around $6.2 \%$ with 2 peaks in June (16.2\%) and November (14.3\%). Monthly incidences of SA clinical samples and infections

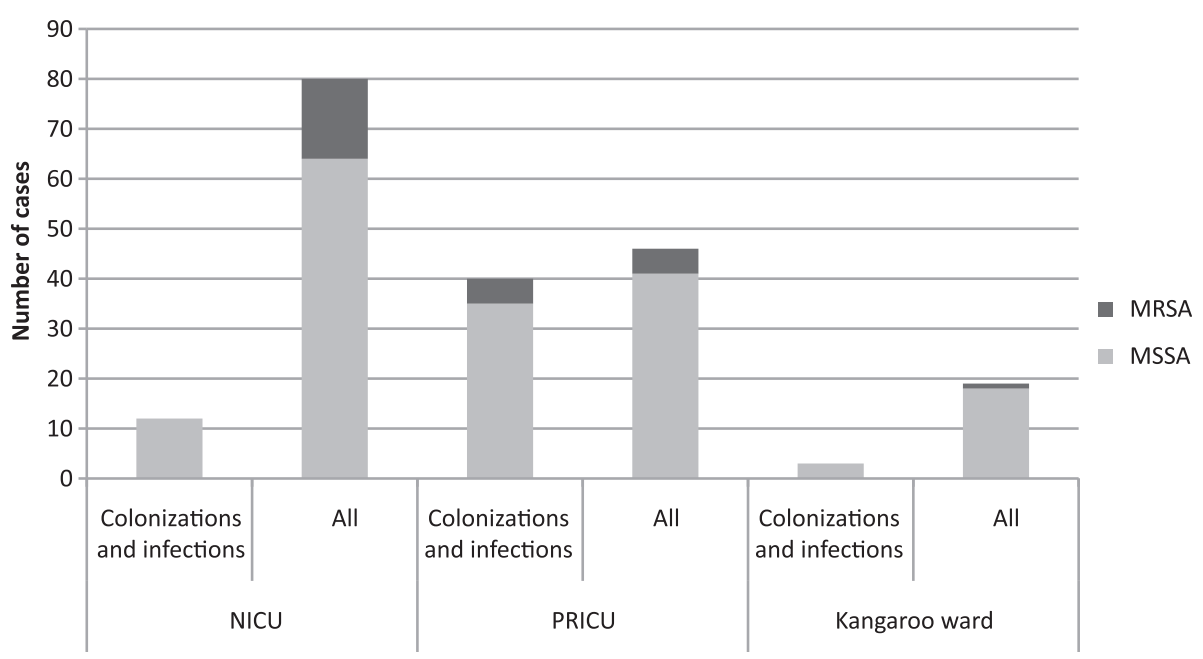

Figure 1 Distribution of SA carriage, colonisation and infection for each sector in 2009. All=carriage, colonization and infection. 
Table 1 Typological analysis of colonization- and infection-associated clinical SA positive samples by care sector

\begin{tabular}{|c|c|c|c|c|c|c|c|c|}
\hline & \multicolumn{2}{|c|}{ NICU } & \multicolumn{2}{|c|}{ PRICU } & \multicolumn{2}{|c|}{ Kangaroo ward } & \multicolumn{2}{|c|}{ Total } \\
\hline & $\mathrm{n}$ & $\%$ & $\mathrm{n}$ & $\%$ & $\mathrm{n}$ & $\%$ & $\mathrm{n}$ & $\%$ \\
\hline \multicolumn{9}{|c|}{ Sites of isolation } \\
\hline Respiratory & 4 & 20 & 39 & 63.9 & 2 & 40 & 45 & 52.3 \\
\hline Ophthalmic & 9 & 45 & 4 & 6.6 & 2 & 40 & 15 & 17.5 \\
\hline Blood & 2 & 10 & 8 & 13.1 & 0 & 0 & 10 & 11.6 \\
\hline Catheter & 2 & 10 & 5 & 8.2 & 0 & 0 & 7 & 8.1 \\
\hline Other & 3 & 15 & 5 & 8.2 & 1 & 20 & 9 & 10.5 \\
\hline Total & 20 & & 61 & & 5 & & 86 & \\
\hline
\end{tabular}

by care sector were also calculated. Evolution of incidences over the year was very similar to prevalence curves, especially in the NICU with increased incidence in March (26.1\%), July (20\%) and August (17.4\%), and November (28.9\%) (data not shown). Finally, epidemiological analyses showed several epidemic episodes on a background of SA endemicity: each care sector presented 2 or 3 prevalence peaks.

Monthly MSSA and MRSA prevalence rates in the entire NCC are summarized in Figure 3. The overall MSSA prevalence did not decrease below 5.5\% (in May and October), and reached $13.2 \%$ in March. Concerning MRSA, the mean prevalence in 2009 for all of the NCC was around $1.53 \%$, with increasing rates in September (3.7\%) and November (6\%). These results show that MSSA endemicity largely exceeded MRSA endemicity in the NCC. Incidence rates confirmed that new cases of MSSA were also more frequent than MRSA in the NCC (data not shown).

Monthly analysis of infections showed an emergence of SA bacteraemia and catheter-linked infections in summer.
For one of them, positive blood-culture was associated with a positive catheter. The origin of the other bacteraemia was not found.

\section{MSSA infections outbreak}

The epidemic curve of SA infection cases between January and December 2009 is shown in Figure 4. Among the 17 SA infections, 15 were caused by MSSA strains versus 2 by MRSA. Outbreak alert was sounded because of an increase in MSSA case numbers observed in July (4 cases) and August (2 cases), contrasting with the average rate of one case every 2 months until then. Six other SA infections occurred in September and October with respectively 3 cases including one MRSA infection each month. Another MSSA infection in November and one in December were also reported.

The 6 cases of July and August led to the outbreak investigation. All 6 patients were hospitalized in the PRICU and 5 of them were preterm neonates. Principal characteristics of these patients and associated strains are given in Table 2. Antimicrobial susceptibility was tested for the 12 MSSA strains from the 6 patients (Table 2). The 12 strains were mostly penicillinase producers $(\mathrm{n}=11)$ and susceptible to most antibiotic classes except one strain resistant to macrolides. Most strains lacked virulence factors since only one carried tst, the gene encoding for toxic shock syndrome toxin 1 and one other carried sea coding for enterotoxin A. PFGE-typing for the 12 strains revealed a predominant profile named pulsotype $\mathrm{C}$ and shared by 8 strains isolated from 4 patients (Table 2). Interestingly, some isolates with the same pulsotype displayed different content in virulence genes.

The synopsis of the IC team's interventions and investigations is presented in Table 3. During the environmental investigation, a high level of surface contamination (109 surfaces sampled) in all areas (average rate of $82.6 \%$ ) of

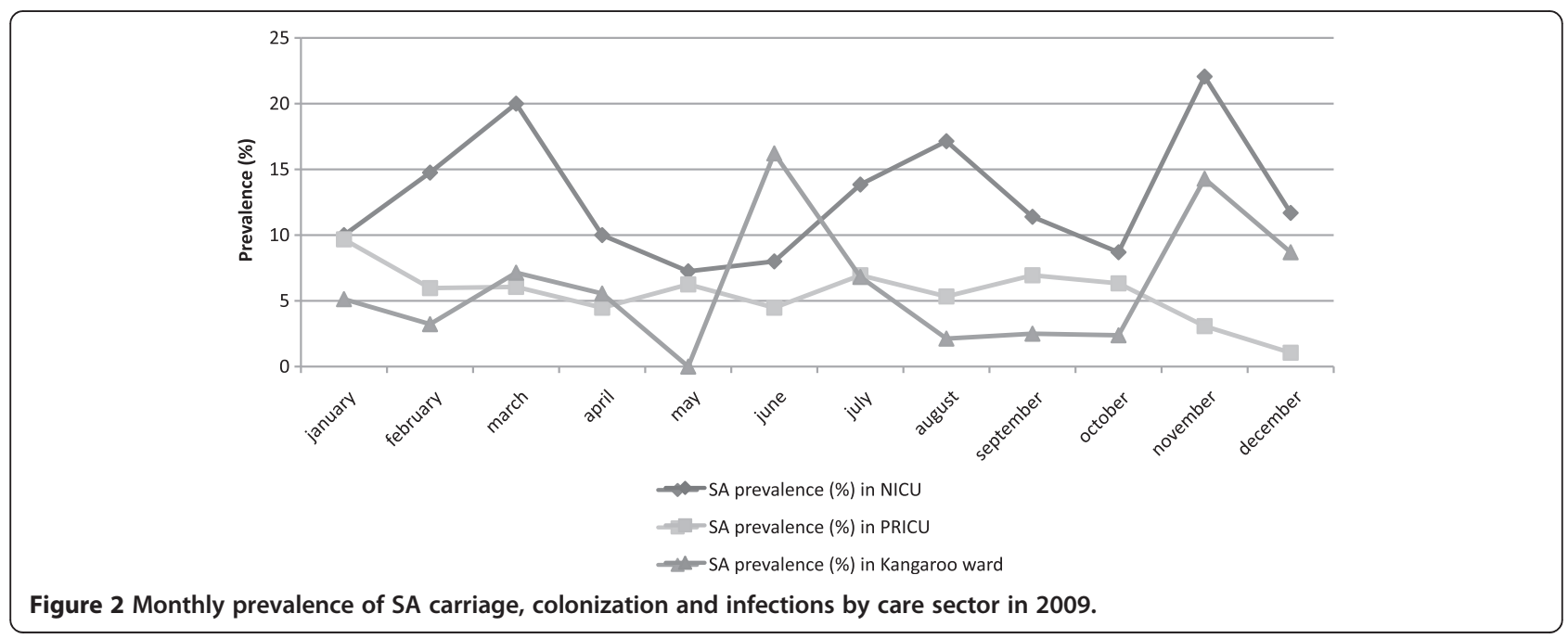




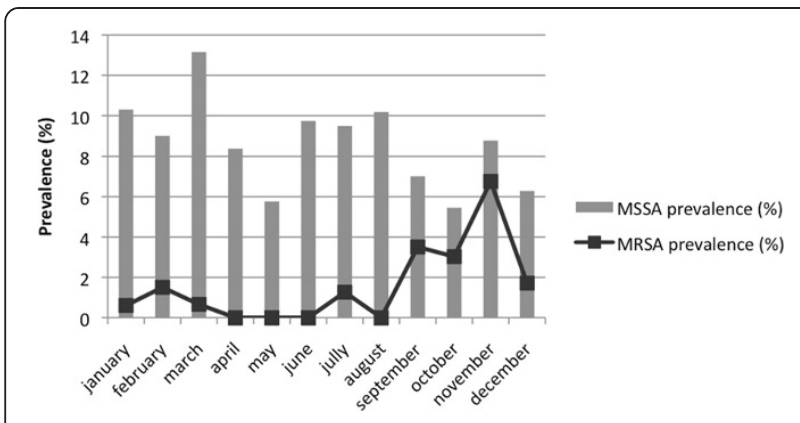

Figure 3 MSSA and MRSA monthly prevalence in 2009 for NCC.

the NCC was observed with the presence of pathogens such as Enterococcus faecalis, Pseudomonas aeruginosa and MSSA. MSSA were isolated from a rack of electric syringes, medical records of patient, 2 spare beds stored in the hallway, and an incubator. All of these environmental MSSA strains were typed by PFGE and differed from the profile C. Water samples $(n=19)$ at various points of use were also analyzed, but none was positive for SA.

The clinical audit of hand hygiene practices revealed compliance rates of $60 \%$ in the NICU (14 persons assessed) and $76 \%$ in the PRICU (16 persons assessed). Noncompliance with recommendations was mostly due to the concatenation of multiple care sequences for the same patient, for example the absence of hand hygiene between a contaminated care (nap change) and a clean one (catheter manipulation). The assessment of central venous catheter manipulations in the PRICU revealed failures in catheter monitoring: catheter insertion points were covered with opaque dressings preventing visual control, and the frequency of dressing changes was insufficient. Furthermore, health-care workers always wore white over-gowns whenever tending to a catheter or coming into direct contact with perfused patients. These gowns were stored in a drawer of the incubator until re-use. During the observations, these white gowns were pointed out as a potential bacterial reservoir or source of cross-contamination. Thereby, 35 of them were sampled in December: 25 gowns in the PRICU and 10 in the NICU. Many of them were positive (15/35) for several pathogens including SA, which was found on 8 separate gowns. Overall these gowns showed a high level of contamination (on average $50 \mathrm{CFU} / 25 \mathrm{~cm}^{2}$ ). SA strains were not typed due to the delay since the summer outbreak but the level of contamination suggested that misuse of these gowns could promote the transmission of pathogens.

\section{Discussion and conclusion}

The study of SA epidemiology in the Montpellier NCC showed an MSSA infection outbreak in the context of SA endemicity. The overall prevalence of SA in 2009 was consistent with other published studies $[11,21]$. The SA outbreak consisted mostly of bloodstream and respiratory tract infections, mainly caused by the same clone in PFGE. Observations by the IC team suggested that slack healthcare practices could be directly linked to the outbreak. Poor practice concerned mainly the manipulation and monitoring of central venous catheters, and the use of non-disposable white gowns. Aside from constant attention to hand hygiene [22,23], the complexity of care in neonatology requires a perfect knowledge of infection control principles, enabling healthcare professionals to cluster interventions by risk level so as to limit selfcontamination of the patient or contamination of his

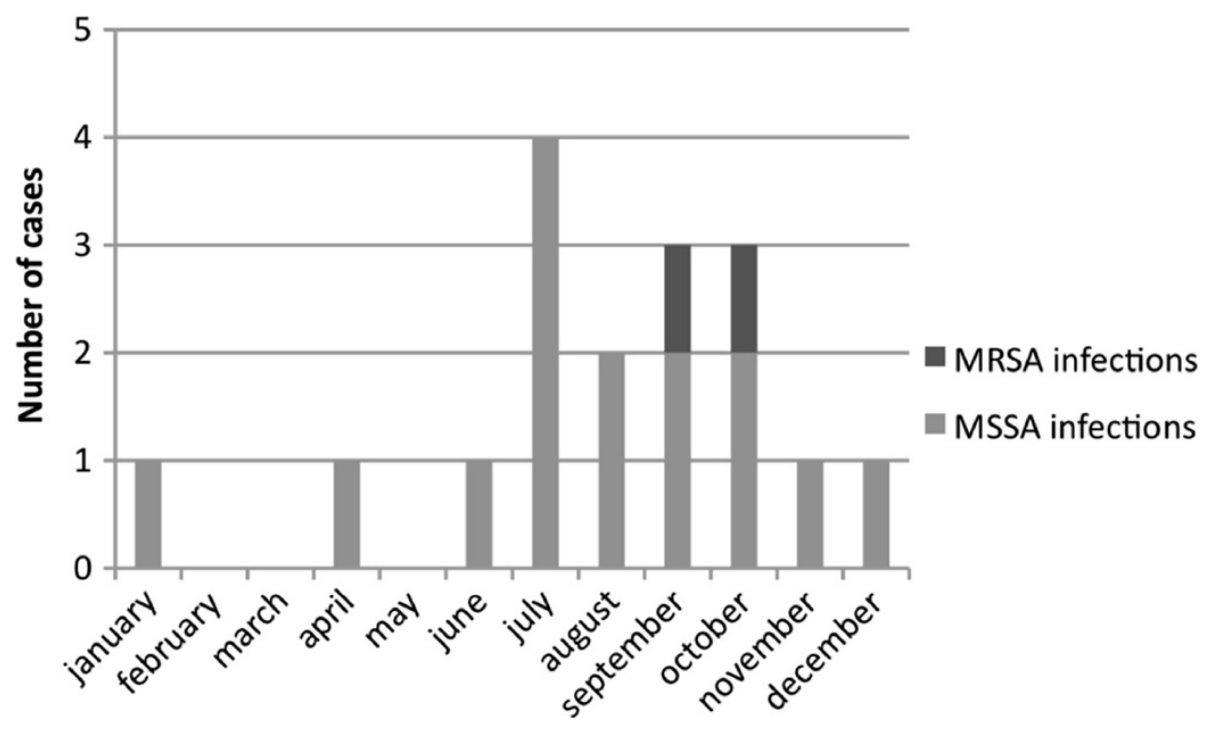

Figure 4 Evolution of SA infections in 2009 in NCC. 
Table 2 Principal clinical characteristics of outbreak patients and strains

\begin{tabular}{|c|c|c|c|c|c|c|c|c|c|c|c|c|}
\hline Patient & Reason of admission & Samples & Nature of sample & NCC sector & PFGE profile & MSSA/MRSA & luk-PV & sea & tst & Penicilline G & Oxacilline & Kanamycin \\
\hline \multirow[t]{2}{*}{1} & Prematurity and NEC & $1 \mathrm{~A}$ & BSI & $\mathrm{NICU}$ & A & MSSA & & & & S & S & S \\
\hline & & 1B & & PRICU & B & MSSA & & & & $R$ & S & S \\
\hline \multirow[t]{2}{*}{2} & Severe epileptic encephalopathy & $2 \mathrm{~A}$ & BSI & PRICU & C & MSSA & & & & $\mathrm{R}$ & S & S \\
\hline & & $2 B$ & Conjunctivitis & & C & MSSA & & + & & $\mathrm{R}$ & S & S \\
\hline \multirow[t]{3}{*}{3} & Prematurity and NEC & $3 \mathrm{~A}$ & Insertion point of $\mathrm{KT}$ & PRICU & C & MSSA & & & & R & S & S \\
\hline & & $3 B$ & KT & & C & MSSA & ND & ND & ND & R & S & S \\
\hline & & $3 C$ & & & C & MSSA & ND & ND & ND & R & S & S \\
\hline \multirow[t]{2}{*}{4} & Prematurity & $4 \mathrm{~A}$ & BSI & PRICU & Untypable & MSSA & & & & R & S & S \\
\hline & & $4 \mathrm{~B}$ & & & Untypable & MSSA & ND & ND & ND & R & S & S \\
\hline \multirow[t]{2}{*}{5} & Prematurity & $5 \mathrm{~A}$ & Tracheal Asp. & PRICU & C & MSSA & & & & $\mathrm{R}$ & S & S \\
\hline & & $5 B$ & $B L$ & & C & MSSA & & & + & R & S & S \\
\hline 6 & Severe epileptic encephalopathy & $6 \mathrm{~A}$ & Tracheal Asp. & PRICU & C & MSSA & & & - & $\mathrm{R}$ & $\mathrm{s}$ & S \\
\hline
\end{tabular}


Table 2 Principal clinical characteristics of outbreak patients and strains (Continued)

\begin{tabular}{|c|c|c|c|c|c|c|c|c|c|c|c|}
\hline Patient & Gentamicin & Tobramycin & Tetracycline & Macrolides & Cotrimoxazole & Fluoroquinolones & Rifamycin & Fusic acid & Fosfomycin & Glycopeptides & Linezolid \\
\hline \multirow[t]{2}{*}{1} & S & S & S & S & S & S & S & S & S & S & S \\
\hline & S & S & S & S & S & S & S & S & S & S & ND \\
\hline \multirow[t]{2}{*}{2} & S & S & S & S & S & S & s & S & S & S & S \\
\hline & S & S & S & S & S & S & $S$ & S & S & S & S \\
\hline \multirow[t]{3}{*}{3} & S & S & S & S & S & S & S & S & S & S & ND \\
\hline & S & S & S & S & S & S & S & S & S & S & ND \\
\hline & S & S & S & S & S & S & S & S & S & S & ND \\
\hline \multirow[t]{2}{*}{4} & S & S & S & S & $S$ & $S$ & $S$ & $S$ & $S$ & $S$ & $S$ \\
\hline & S & $S$ & $S$ & S & $S$ & $S$ & $S$ & $S$ & S & $S$ & $S$ \\
\hline \multirow[t]{2}{*}{5} & $S$ & $S$ & $S$ & $S$ & $S$ & $S$ & $S$ & $S$ & $S$ & $S$ & ND \\
\hline & $S$ & $S$ & $S$ & R & $S$ & $S$ & $S$ & $S$ & $S$ & S & S \\
\hline 6 & S & S & $S$ & $S$ & S & $S$ & $S$ & S & $S$ & S & S \\
\hline
\end{tabular}

NEC: necrotizing enterocolitis, BSI: bloodstream infection, KT: catheter, Asp.: aspiration, BL: bronchial liquid, luk-PV, gene coding for Panton-Valentine leukocidin; sea, genes coding for staphylococcal enterotoxins $\mathrm{A}$; tst, gene coding for toxic shock syndrome toxin $1, N D$, non determinate. 
Table 3 Outbreak investigation and IC interventions

\begin{tabular}{ll}
\hline Actions & Dates \\
\hline Case study & August 07, 17 and 18, 2009 \\
- Clinical records review and & \\
strains typing & \\
Environmental investigation & $\begin{array}{l}\text { August 05, 12, September 15 } \\
\text { and October 13, 2009 } \\
\text { - Surface and water sampling }\end{array}$ \\
- Gowns & December 2009 (retrospectively) \\
Hygiene practices assessment & October 01, 2009 \\
- Hand hygiene & October 19, 24 and \\
- Catheter-linked practices & November 09, 2009 \\
\hline
\end{tabular}

environment $[22,23]$. Some studies have confirmed the progressive contamination of hands or gloves [24] and identified key opportunities for hand hygiene during routine cares, even if wearing gloves, because of possible hand contamination during removal [22]. Moreover, health care workers' white coats remain a controversial subject, supposedly protecting the patient during central venous catheter manipulations, but highly contaminated by pathogens for most of them (about a quarter positive for SA). As previously described by Treakle et al. in 2009, we could imagine that they were a vector of patient-to-patient transmission, relaying SA circulation and outbreak in the NCC [25]. However, we did not investigate the possibility of SA chronic carriage by a health care worker which could also have relayed the outbreak $[8,26]$.

The typological analysis of colonized sites and infections occurring in 2009 showed a majority of cases in the PRICU, where patients are most susceptible to infections. The occurrence of catheter-related infections in the summer was consistent with the increased SA prevalence (colonization pressure), and slack catheter monitoring. Catheter-related infections are the most common healthcare associated infections in NICUs $[22,27,28]$. The general strategy for their prevention is based on good practice recommendations concerning (i) insertion and maintenance of indwelling lines, (ii) administration of prophylactic antibiotics e.g.: antibiotic lock therapy, (iii) use of skin emollients to reduce bacterial penetration, and (iv) healthcare workers and visitors donning of single-use gowns [22]. However in our NCC, points (ii) and (iii) were not applied and compliance with points (i) and (iv) was not optimal.

SA is a significant pathogen in neonatology and an important cause of morbidity [7]. Epidemiological studies of MRSA in NICUs have reported widely varying prevalence rates, ranging from 0.6 to $53 \%[6,7,21,29,30]$, and MRSA outbreaks are often described [6,31]. Far less attention is given to MSSA. In the Montpellier NCC, MRSA prevalence appeared rather low $(1.53 \%$ in 2009 , ranging from 0 to $6.1 \%$ ), and quantitatively a less important problem than
MSSA. We believe the commonly encouraged focus on MRSA surveillance $[22,27,28]$ may lead to unrecognized or underestimated spread of MSSA. This is particularly worrying for infection control in wards where MSSA is the most prevalent SA type, as we observed for the NCC.

\section{Abbreviations \\ CoNS: Coagulase-negative staphylococci; HAl: Health-care associated infections; IC: Infection control; luk-PV: gene encoding the panton-valentine leukocidin; MDRB: Multi-drug resistant bacteria; MRSA: Methicillin-resistant Staphylococcus aureus; MSSA: Methicillin-susceptible Staphylococcus aureus; NCC: Neonatal care center; NICU: Neonatal intensive care unit; PFGE: Pulsed-field gel electrophoresis; PRICU: Pediatric reanimation and intensive care unit; PVL: Panton-valentine leukocidin; SA: Staphylococcus aureus; sea gene: Encoding the staphylococcal enterotoxins A; tst gene: Encoding the toxic shock syndrome toxin 1.}

\section{Competing interests}

The authors declare that they have no competing interests.

\section{Authors' contributions}

$\mathrm{SRB}, \mathrm{AF}, \mathrm{RM}, \mathrm{MND}$ and $\mathrm{GC}$ participated in the data collection. SRB and AF contributed to bacteriological and epidemiologic analysis. SRB, AL, LB, EJB and SP contributed to outbreak investigation and data interpretation. SRB and AF wrote the manuscript. All authors read and approved the final manuscript.

\section{Acknowledgements}

We thank all of the IC and NCC teams for their participation in the study.

\section{Author details}

'Université Montpellier 1, UMR 5119, Equipe Pathogènes et Environnements, U.F.R. des Sciences Pharmaceutiques et Biologiques, 15, Avenue Charles Flahault, BP 14491, 34093 Montpellier Cedex 5, France. ${ }^{2}$ Centre Hospitalier Régional Universitaire de Montpellier, Hôpital La Colombière, Service d'Hygiène Hospitalière, 39 avenue Charles Flahault, 34295 Montpellier Cedex 5, France. ${ }^{3}$ Centre Hospitalier Régional Universitaire de Nîmes, Service de Pédiatrie, Hôpital Caremeau, Place du Pr R. Debré, 30029 Nîmes Cedex 9, France. ${ }^{4}$ Centre Hospitalier Régional Universitaire de Montpellier, Service de Néonatologie, Hôpital Arnaud de Villeneuve, 371 Avenue du Doyen Gaston Giraud, 34295 Montpellier Cedex 5, France. ${ }^{5}$ Centre Hospitalier Régional Universitaire de Montpellier, Laboratoire de Bactériologie, Hôpital Arnaud de Villeneuve, 371 Avenue du Doyen Gaston Giraud, 34295 Montpellier Cedex 5, France.

Received: 3 March 2014 Accepted: 25 May 2014

Published: 1 July 2014

\section{References}

1. Jacquot A, Neveu D, Aujoulat F, Mercier G, Marchandin H, Jumas-Bilak E, et Picaud J-C: Dynamics and clinical evolution of bacterial gut microflora in extremely premature patients. J Pediatr 2011, 158(3):390-396.

2. Marchant EA, Boyce GK, Sadarangani M, et Lavoie PM: Neonatal sepsis due to coagulase-negative staphylococci. Clin Dev Immunol 2013, 2013:586076.

3. Jeong IS, Jeong JS, et Choi EO: Nosocomial infection in a newborn intensive care unit (NICU), South Kore. BMC Infect Dis 2006, 6:103.

4. Healy CM, Palazzi DL, Edwards MS, Campbell JR, et Baker CJ: Features of invasive staphylococcal disease in neonates. Pediatrics 2004, 114(4):953-961.

5. Giuffrè $M$, Bonura $C$, Cipolla D, et Mammina C: MRSA infection in the neonatal intensive care unit. Expert Rev Anti Infect Ther 2013, 11(5):499-509.

6. Nübel U, Nachtnebel M, Falkenhorst G, Benzler J, Hecht J, Kube M, Bröcker F, Moelling K, Bührer C, Gastmeier P, Piening B, Behnke M, Dehnert M, Layer F, Witte W, et Eckmanns T: MRSA transmission on a neonatal intensive care unit: epidemiological and genome-based phylogenetic analyses. PLoS One 2013, 8(1)::54898.

7. Nelson MU, et Gallagher PG: Methicillin-resistant Staphylococcus aureus in the neonatal intensive care unit. Semin Perinatol 2012, 36(6):424-430. 
8. Geva A, Wright SB, Baldini LM, Smallcomb JA, Safran C, et Gray E: Spread of methicillin-resistant Staphylococcus aureus in a large tertiary NICU: network analysis. Pediatrics 2011, 128(5):e1173-1180.

9. van der Mee-Marquet N, François P, Domelier A-S, Arnault L, Girard N, Schrenzel J, Quentin R, et Bloodstream Infection Study Group of the Réseau des Hygiénistes du Centre: Variable-number tandem repeat analysis and multilocus sequence typing data confirm the epidemiological changes observed with Staphylococcus aureus strains isolated from bloodstream infections. J Clin Microbiol 2009, 47(9):2863-2871.

10. van der Mee-Marquet N, Epinette C, Loyau J, Arnault L, Domelier A-S, Losfelt B, Girard N, Quentin R, et Bloodstream Infection Study Group of the Relais d'Hygiène du Centre: Staphylococcus aureus strains isolated from bloodstream infections changed significantly in 2006. J Clin Microbiol 2007, 45(3):851-857.

11. Graham PL 3rd, Morel A-S, Zhou J, Wu F, Della-Latta P, Rubenstein D, et Saiman L: Epidemiology of methicillin-susceptible Staphylococcus aureus in the neonatal intensive care unit. Infect Control Hosp Epidemiol 2002, 23(11):677-682.

12. Weist $K$, Wendt $C$, Petersen $L R$, Versmold $H$, et Rüden $H$ : An outbreak of pyodermas among neonates caused by ultrasound gel contaminated with methicillin-susceptible Staphylococcus aureus. Infect Control Hosp Epidemiol 2000, 21(12):761-764.

13. Wilcox MH, Fitzgerald P, Freeman J, Denton M, Gill AB, Hoy C, Parnell P, Porter C, Haspinall $L$, et Hawkey P: A five year outbreak of methicillin-susceptible Staphylococcus aureus phage type 53,85 in a regional neonatal unit. Epidemiol Infect 2000, 124(1):37-45.

14. Boers SA, van Ess I, Euser SM, Jansen R, Tempelman FRH, et Diederen BMW: An outbreak of a multiresistant methicillin-susceptible Staphylococcus aureus (MR-MSSA) strain in a burn centre: the importance of routine molecular typing. Burns 2011, 37(5):808-813.

15. Lindqvist M, Isaksson B, Samuelsson A, Nilsson LE, Hallgren A: A clonal outbreak of methicillin-susceptible Staphylococcus aureus with concomitant resistance to erythromycin, clindamycin and tobramycin in a Swedish county. Scand J Infect Dis 2009, 41(5):324-333.

16. Acton DS, Plat-Sinnige MJT, van Wamel W, de Groot N, et van Belkum A: Intestinal carriage of Staphylococcus aureus: how does its frequency compare with that of nasal carriage and what is its clinical impact? Eur J Clin Microbiol Infect Dis 2009, 28(2):115-127.

17. Bhalla A, Aron DC, et Donskey CJ: Staphylococcus aureus intestinal colonization is associated with increased frequency of $\mathrm{S}$. aureus on skin of hospitalized patients. BMC Infect Dis 2007, 7:105.

18. Durand S, Rideau Batista Novais A, Mesnage R, Combes C, Didelot M-N, Lotthé A, Filleron A, Baleine J, et Cambonie G: Validation of Nosocomial Infection in Neonatology: A new Method for Standardized Surveillance. 2014. In press.

19. Predari SC, Ligozzi M, et Fontana R: Genotypic identification of methicillin-resistant coagulase-negative staphylococci by polymerase chain reaction. Antimicrob Agents Chemother 1991, 35(12):2568-2573

20. Corne $\mathrm{P}$, Marchandin H, Jonquet O, Campos J, et Bañuls A-L: Molecular evidence that nasal carriage of Staphylococcus aureus plays a role in respiratory tract infections of critically ill patients. J Clin Microbiol 2005, 43(7):3491-349.

21. Kuo C-Y, Huang Y-C, Huang DT-N, Chi H, Lu C-Y, Chang L-Y, Chi C-Y, Ho Y-H, Huang Y-C, Liu C-C, et Huang L-M: Prevalence and Molecular Characterization of Staphylococcus aureus Colonization among Neonatal Intensive Care Units in Taiwan. Neonatology 2013, 105(2):142-148.

22. Polin RA, Denson S, Brady MT, Committee on Fetus and Newborn, et Committee on Infectious Diseases: Strategies for prevention of health care-associated infections in the NICU. Pediatrics 2012, 129(4):1085-1093.

23. Lam BCC, Lee J, et Lau YL: Hand hygiene practices in a neonatal intensive care unit: a multimodal intervention and impact on nosocomial infection. Pediatrics 2004, 114(5):565-571.

24. Pessoa-Silva CL, Dharan S, Hugonnet S, Touveneau S, Posfay-Barbe K, Pfister R, et Pittet $D$ : Dynamics of bacterial hand contamination during routine neonatal care. Infect Control Hosp Epidemiol 2004, 25(3):192-197.

25. Treakle AM, Thom KA, Furuno JP, Strauss SM, Harris AD, et Perencevich EN: Bacterial contamination of health care workers' white coats. Am J Infect Control 2009, 37(2):101-105.

26. Bertin ML, Vinski J, Schmitt S, Sabella C, Danziger-Isakov L, McHugh M, Procop GW, Hall G, Gordon SM, et Goldfarb J: Outbreak of methicillin-resistant Staphylococcus aureus colonization and infection in a neonatal intensive care unit epidemiologically linked to a healthcare worker with chronic otitis. Infect Control Hosp Epidemiol 2006, 27(6):581-585.

27. Polin RA, et Saiman L: Nosocomial infections in the neonatal intensive care unit. Neoreviews 2003, 4(3):e81-e89.

28. Polin RA, Denson S, Brady MT, Committee on Fetus and Newborn, et Committee on Infectious Diseases: Epidemiology and diagnosis of health care-associated infections in the NICU. Pediatrics 2012, 129(4):e110-1109.

29. Huang Y-C, Chou Y-H, Su L-H, Lien R-I, et Lin T-Y: Methicillin-resistant Staphylococcus aureus colonization and its association with infection among infants hospitalized in neonatal intensive care units. Pediatrics 2006, 118(2):469-474.

30. Morioka I, Takahashi N, Kitajima H, t committee for infection prevention and vaccine promotion of the Japan Society for Premature and Newborn Medicine: Prevalence of MRSA-colonized patients in Japanese neonatal care units in 2011. Pediatr Int 2013, 56(2):211-214

31. Saiman L, Cronquist A, Wu F, Zhou J, Rubenstein D, Eisner W, Kreiswirth BN, et Della-Latta P: An outbreak of methicillin-resistant Staphylococcus aureus in a neonatal intensive care unit. Infect Control Hosp Epidemiol 2003, 2(5):317-321.

doi:10.1186/2047-2994-3-21

Cite this article as: Romano-Bertrand et al:: Staphylococcus aureus in a neonatal care center: methicillin-susceptible strains should be a main concern. Antimicrobial Resistance and Infection Control 2014 3:21.

\section{Submit your next manuscript to BioMed Central and take full advantage of:}

- Convenient online submission

- Thorough peer review

- No space constraints or color figure charges

- Immediate publication on acceptance

- Inclusion in PubMed, CAS, Scopus and Google Scholar

- Research which is freely available for redistribution

Submit your manuscript at www.biomedcentral.com/submit
C Biomed Central 